\title{
Web Pages Ranking Algorithms: A Survey
}

\author{
Ayad Abdulrahman Saleem \\ Information Technology Department \\ Duhok Polytechnic University \\ Computer Science Department \\ University of Zakho \\ Zakho, Iraq \\ ayad.abdulrahman@dpu.edu.krd \\ https://doi.org/10.48161/qaj.v1n3a79
}

\begin{abstract}
Due to the daily expansion of the web, the amount of information has increased significantly. Thus, the need for retrieving relevant information has also increased. In order to explore the internet, users depend on various search engines. Search engines face a significant challenge in returning the most relevant results for a user's query. The search engine's performance is determined by the algorithm used to rank web pages, which prioritizes the pages with the most relevancy to appear at the top of the result page. In this paper, various web page ranking algorithms such as Page Rank, Time Rank, EigenRumor, Distance Rank, SimRank, etc. are analyzed and compared based on some parameters, including the mining technique to which the algorithm belongs (for instance, Web Content Mining, Web Structure Mining, and Web Usage Mining), the methodology used for ranking web pages, time complexity (amount of time to run an algorithm), input parameters (parameters utilized in the ranking process such as InLink, OutLink, Tag name, Keyword, etc.), and the result relevancy to the user query.
\end{abstract}

Keywords - Search Engines, Ranking Algorithms, WCM, Web Mining, HITS, Crawler.

\section{INTRODUCTION}

Generally, the World Wide Web (WWW) is a collection of hypertext documents that are connected together $[1,2]$. The WWW presents an architectural design for gaining access to documents linked that are widespread across millions of computers on the Internet [3-5]. Obtaining valuable information from the immense volume of information on the internet was one of the most difficult undertakings. Web search engines have emerged as a handy tool for searching for intended valuable content on the WWW by utilizing user-supplied search phrases [6]. In general, the results of the search process for the search engine are shown in the form of a list, which is often described as the search engine result page (SERP) [7]. The search engine retrieves the results of the search in a form of a jumble of irrelevant and relevant information [8]. To obtain any information on the web [9], the user uses the preferred search engine, enters a word or phrase to ask for a query, and therefore clicks on the retrieved results. No user can browse all web pages retrieved in response to the query requested by the user. As a consequence, search engines assist users in locating the most relevant web pages worth visiting by showing the resulting pages in a ranked order determined by various page rank algorithms [8, 10]. The traditional search engine technology may be generally categorized into two types [11]: human-powered directorybased engines and crawler-based engines. The humanspowered directory, such as the Open Directory, relies on humans to maintain its lists. In this setting, web pages are saved in various directories according to their type. Whenever a query is executed, it is first classified, and then the suitable directory is searched for the relevant web page. They are created when a website's owner sends the site for the reviewing along with a brief description of the website [11]. Generally, a search is limited to matches within the submitted descriptors.

Web crawling, also known as a crawler or spider, is a process of downloading and storing web pages, most frequently for the purpose of indexing them in a search engine [12]. Generally, a crawler begins by adding an initial set of Uniform Resource Locators (URLs) to a queue, which stores and prioritizes all URLs to be retrieved [13]. The crawler retrieves URLs from this queue, downloads the pages, extracts any URLs included within the downloaded page, and adds the extracted URLs to the queue [3,14]. This process's repetition is continued until the crawler decides to finish. The pages collected are eventually utilized for various purposes, such as web search engines. By automating the process of link traversal, Web Crawler facilitates users in their Web navigation. A web crawler generates a set of web pages that were searched and indexed by a search engine in order to satisfy the user's query. A user 
submits a query to a precomputed index, which returns a list of documents matching the query [3]. In fact, the web crawler is the most critical component of a search engine, as it plays a critical role in retrieving the information.

Algorithms for ranking web pages have a role in the last component. The exact information wanted by the user is unpredictable [15]. Thus, ranking algorithms of the web pages are created to expect the requirements of the user based on a variety of dynamic (such as popularity) and static (such as textual content and hyperlinks count) characteristics. These are critical aspects that contribute to the superiority of one search engine over another [15]. Ranking algorithms are critical for web pages' ranking in order to receive the best results that are the most relevant to the query requested by the user [16]. The search engine typical process is showed in Figure 1, which displays the block diagram of the user's search query.

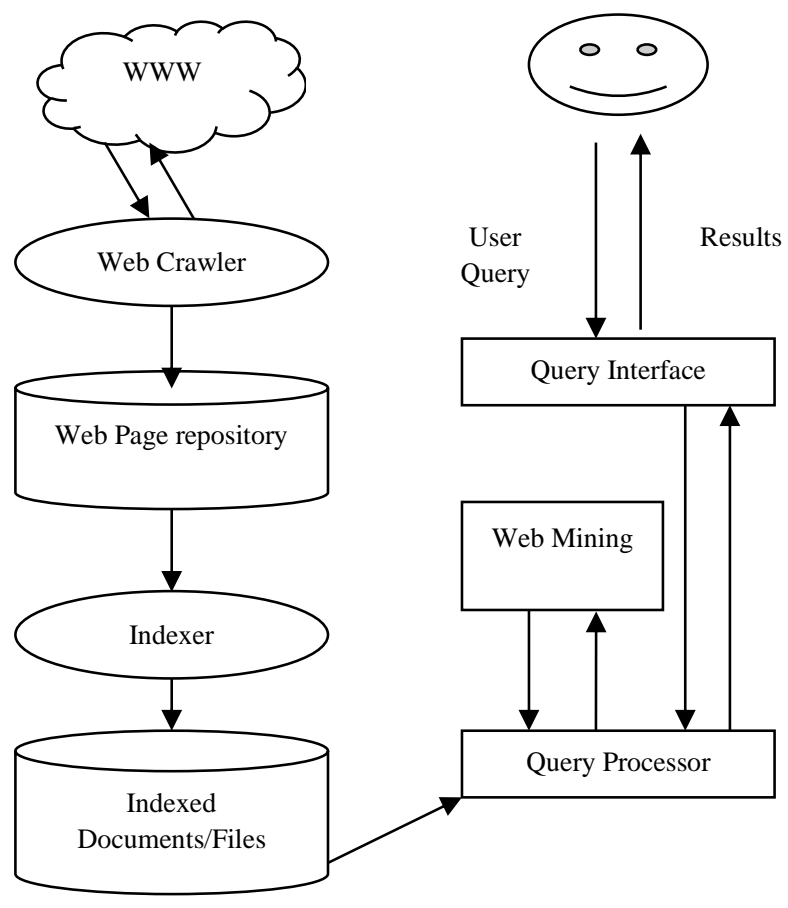

Figure 1: Search engine working process [14], [17]

The aim of this paper is to provide an analysis for common various algorithms of ranking web pages, such as Page Rank, Hyperlink Induced Topic Search Algorithm (HITS), Weighted Page Rank (WPR), Weighted Link Rank (WLR), EigenRumor, Distance Rank, Time Rank, Relation based, SimRank, Query Dependent Ranking, Tag Rank, and Dirichlet Rank algorithm.

\section{WEB MINING}

Hyperlink Analyze is a portion of a larger study domain known as Web Mining, which is defined as the process of utilizing techniques of data mining in order for extracting valuable information from data on the Web [18]. Web mining assists the users of the internet about upcoming web pages to be visited later [19]. Three types of data, that may be gathered and utilized for analyzing Web Mining, including usage data, content data, and structural data. Based on the type of data to be mined [20], web mining may be classified into the following three main categories:

1) Web Usage Mining (WUM):- This method discovers an internet user's usage history of the visited web pages. A $\log$ file is generated for each user of the internet and gives a higher weight to web pages that are most visited by the users of the internet [17]. The most common utilization data gathered on a web page [18] includes the users' access times, IP addresses, and page references.

2) Web Content Mining (WCM):- WCM is in charge of searching for the most relevant and accurate information from web pages' contents on the internet [19]. The content data refers to the collection of facts that a webpage was created to provide to its users. It may be composed of photos, text, video, and music, as well as organized data in form of tables and lists [18].

3) Web Structure Mining (WSM):- This method explores WWW by taking into account the web pages' hyperlinks. It can give additional weight to web pages that have significant and valuable links for example ones that have a larger number of OutLinks. In addition, the web pages that have a larger number of InLinks can be considered the most significant if they are linked with a larger number of other websites [17].

Web mining employs a variety of techniques such as clustering, association rules, and classification to extract data from WWW [17]. Clustering methods include gridbased clustering, clustering by agglomerative methods, partitioning clustering, model-based clustering, and densitybased clustering [21-26]. Association rules involve some techniques such as quantitative, multilevel, and multidimensional. While the classification methods used are the Bayesian classification technique, Decision Tree (DT), Support Vector Machine (SVM), and Artificial Neural Networks (ANN).

\section{RANKING ALGORITHMS}

Due to the massive amount of information on the internet, some approaches are required to retrieve the information relevant to the user query. The mechanism for retrieving the information from the internet is strongly depending on the search engine [27]. A search engine's primary role is to provide relevant and accurate results to an internet user's query. The search engine ranks pages descending based on the most relevant by using a variety of ranking algorithms [28]. The ranking of a web page for a particular query is based on a variety of factors, such as the relevancy to the concepts and words contained in the user 
query, its link popularity, and so on. Numerous ranking algorithms assess the relevance of websites depending on their content (web content mining), while others are depending on the web page popularity (web structure mining), and yet others combine both structure and content mining to determine the web page's relevancy. The various algorithms that are used to rank web pages are explained below:

\section{PageRank Algorithm (PR)}

It is the most popular algorithm used for computing web page ranking. It ranks web pages based on their InLinks and OutLinks. A certain web page is considered important when it has multiple InLinks. In other words, the greater number of OutLinks to a certain webpage, the more significant the webpage. This technique initially determines the count of OutLinks and InLinks on the page in order to calculate the ranking of that page [29].

\section{Weighted Page Rank Algorithm (WPR)}

It is an improved version of PR algorithm. The page rank is calculated through determining the popularity of the page. The web page's popularity is determined by counting InLinks and OutLinks on the page. WPR doesn't distribute the web page rank uniformly across its OutLinks. WPR algorithm presents page rank to all OutLinks web pages based on the webpage popularity [30].

\section{Hyperlink Induced Topic Search Algorithm (HITS)}

This algorithm determines the rank of a web page based on its popularity. Additionally, it computes a web page's InLinks and OutLinks. This algorithm is suggested by Jon Kleinberg, which divides web pages into two types, namely Hub and Authorities [31]. The term "Hub" refers to a page that has links to several web pages. While the term "Authority" refers to a webpage that is pointed to by other pages. The HIT-based algorithm works by determining the popularity of a web page in order to calculate its rank. The popularity of a web page is evaluated by calculating its input (Authority) and output (Hub) links.

\section{EigenRumor Algorithm}

Due to the fast increase in the number of blogging sites, producing a ranking for a blog is becoming increasingly challenging. If both PR and HITS algorithms are applied to blogs directly, the rank of each blog is determined by the PR algorithm, which does not allow for the provision of blogs based on their importance. To address this issue, K. Fujimura et al. [32] introduced the EigenRumor algorithm in order to rank the blogs. This Algorithm assigns a rank to each blog based on the weights of the bloggers' authority and hub, as determined by the eigenvector computation.

\section{Distance Ranking Algorithm}

This method [33] is suggested by A. M. ZarehBidoki and N. Yazdani, based on reinforcement learning that takes the logarithmic distance between web pages into account. This method ranks pages according to the smallest logarithmic distance between different pages.

\section{Query-Dependent Ranking}

In this approach, Lee et al. [34] Make utilization of user queries to improve page ranking algorithm's performance. A component dedicated to computing the similarity among user queries has been integrated into the page ranking algorithm. The algorithm analyzes the similarities of user queries and then utilizes this information to determine the final results to be retrieved for the query of the user. Frequently, the same queries are repeated by the same or various search engines. This method utilizes this information smartly to calculate the ranking score of pages and to increase the accuracy and recall values, which imply higher quality.

\section{SimRank Algorithm}

A novel methodology based on the vector space model was suggested, which utilizes the similarity from the vector space model to determine the web page's rank. This technique [35] efficiently gives a rank to the pages to be fetched by the search engine. The majority of conventional page rank algorithms determine the page rank based on the link structure of the pages, and some of them completely disregard the content of the pages. While the SimRank algorithm takes the content of web pages into account when determining a web page's overall rank score.

\section{Weighted Link Rank Algorithm (WLR)}

With the assistance of a PR algorithm, WLR was developed by R. Baeza and E. Davis [36]. This technique calculates the weight of a link based on three separate parameters: the tags, the length of the anchor text, and the relative location within the web page.

\section{Relation Based Algorithm}

A semantic web-based page ranking algorithm was presented by Lamberti et al. [37] for search engines. The semantic web is an extremely beneficial tool that has been utilized to improve the performance of the webpage ranking algorithm [38-42]. This algorithm is utilized by the search engines that are based on a semantic web in order to enhance the efficiency of the ranking algorithms. The algorithm makes use of data from user searches and associated information in order to increase a web page's rank score. This web page rank algorithm was discovered to be low in terms of time complexity and to deliver excellent accuracy in the results of the user search. Additionally, this method was enhanced further by including the scalability of semantic web-based page repositories. 


\section{Tag Rank Algorithm}

A social annotations-based algorithm has been proposed by Jie et al. [43] in order to rank the web pages. This method measures the tags' heat via utilizing the time factor associated with the new data source tag and the user's annotating activity. In addition, it improves the authentication process for web page ranking.

\section{Time Rank Algorithm}

This method was presented by H. Jiang [44] in order for enhancing the rank score of a web page by utilizing the duration of a visit. This technique makes use of WUM to identify the level of significance of a page for the user. This method adds the visiting duration to the PR algorithm's computed score of the web page. If a page's content is relevant to the user query, the user will stay on the page for a long period of time; otherwise, the user will exit fast, resulting in a brief visiting duration to the page.

\section{Dirichlet Rank}

This method [17] was developed to calculate the page rank of the web pages based on their trusted information and utilizes them in the page ranking of the web page. This algorithm determines the quantitative vertex ranking of a subset of nodes and utilizes it to rank pages. It classifies certain nodes as trusted and others as less trusted or mistrustful. This trust-based page ranking algorithm enhances the page ranking module's quality.

TABLE 1: A COMPARISON BETWEEN DIFFERENT WEB PAGE RANKING ALGORITHMS

\begin{tabular}{|c|c|c|c|c|c|}
\hline Algorithm & $\begin{array}{l}\text { Technique } \\
\text { used }\end{array}$ & Methodology & $\begin{array}{l}\text { Time } \\
\text { complexity }\end{array}$ & Input parameters & Result relevancy \\
\hline PR & WSM & $\begin{array}{l}\text { The page's rank is computed based on the number of } \\
\text { InLinks and OutLinks. }\end{array}$ & $\mathrm{O}(\log \mathrm{n})$ & $\begin{array}{l}\text { InLinks and } \\
\text { OutLinks }\end{array}$ & Low relevancy \\
\hline WPR & WSM & $\begin{array}{l}\text { The page rank is calculated by assigning a weight to a web } \\
\text { page. }\end{array}$ & $\begin{array}{l}\text { Less than } \\
\mathrm{O}(\log \mathrm{n})\end{array}$ & $\begin{array}{l}\text { InLinks and } \\
\text { OutLinks }\end{array}$ & Higher than PR \\
\hline HITS & $\begin{array}{l}\text { WSM, } \\
\text { WCM }\end{array}$ & Calculates the Authorities and Hubs. & $\begin{array}{l}\text { Less than } \\
\mathrm{O}(\log n)\end{array}$ & Logs history & $\begin{array}{l}\text { Moderate } \\
\text { relevancy }\end{array}$ \\
\hline EigenRumor & WCM & $\begin{array}{l}\text { Uses extra parameters to improve the algorithm of } \\
\text { weighted page rank. }\end{array}$ & $\begin{array}{l}\text { Less than } \\
\mathrm{O}(\log n)\end{array}$ & $\begin{array}{l}\text { InLinks, OutLinks, } \\
\text { and Blogs }\end{array}$ & $\begin{array}{l}\text { Higher than PR, } \\
\text { HITS, and WPR }\end{array}$ \\
\hline $\begin{array}{l}\text { Distance } \\
\text { Rank }\end{array}$ & WSM & $\begin{array}{l}\text { Compute the rank of the web page based on reinforcement } \\
\text { learning, which takes into account the logarithmic distance } \\
\text { of the web pages. }\end{array}$ & $\mathrm{O}(\log n)$ & $\begin{array}{l}\text { The distance } \\
\text { between pages }\end{array}$ & $\begin{array}{l}\text { Moderate } \\
\text { relevancy }\end{array}$ \\
\hline $\begin{array}{l}\text { Query- } \\
\text { Dependent }\end{array}$ & WCM & $\begin{array}{l}\text { Utilizes the similarity among the queries to compute the } \\
\text { rank of the page. }\end{array}$ & - & Training query & High relevancy \\
\hline SimRank & WCM & $\begin{array}{l}\text { Utilizes the similarity among the queries to compute the } \\
\text { rank of the page. }\end{array}$ & - & Training query & High relevancy \\
\hline WLR & WSM & $\begin{array}{l}\text { Assigns weight on the basis of relative position, OutLinks, } \\
\text { and InLinks in the page. }\end{array}$ & - & $\begin{array}{l}\text { Link structure and } \\
\text { content }\end{array}$ & - \\
\hline $\begin{array}{l}\text { Relation } \\
\text { Based }\end{array}$ & WSM & $\begin{array}{l}\text { A semantic-based search engine would take keywords into } \\
\text { consideration and retrieve pages only when both keywords } \\
\text { are existed on the page and are relevant to the associated } \\
\text { concept as defined in the relational note of each page. }\end{array}$ & - & Keywords & High relevancy \\
\hline Tag Rank & WCM & $\begin{array}{l}\text { Utilizes visiting duration for ranking the page. The } \\
\text { sequential clicking is utilized sequence vector. }\end{array}$ & $\begin{array}{l}\text { Less than } \\
\mathrm{O}(\log n)\end{array}$ & $\begin{array}{l}\text { Popular tags on } \\
\text { the page, InLinks, } \\
\text { and OutLinks }\end{array}$ & Low relevancy \\
\hline Time Rank & WUM & $\begin{array}{l}\text { Visiting duration is used with the computational score of } \\
\text { the original page rank algorithm. }\end{array}$ & - & $\begin{array}{l}\text { Server logs and } \\
\text { Page Rank }\end{array}$ & High relevancy \\
\hline Dirichlet & $\begin{array}{l}\text { WSM, } \\
\text { WCM }\end{array}$ & $\begin{array}{l}\text { Computes the rank of the page based on link attributes: } \\
\text { anchor text length, tag where a link is contained, position } \\
\text { on the web page. }\end{array}$ & $\mathrm{O}(\log \mathrm{n})$ & $\begin{array}{l}\text { Tag name, } \\
\text { position, and } \\
\text { anchor text }\end{array}$ & Medium \\
\hline
\end{tabular}

\section{DISCUSSION}

In general, web page ranking algorithms can be divided into three main categories based on web mining techniques: WCM, WSM, and WUM. The first category takes the content of the web page into consideration, while WSM ignores the content and takes the structure of the web page, including InLinks and OutLinks. The third category, which is WUM depends on the usage history of the visited web page. Logically, the content of the page can not be ignored and should be considered in order to give better results with higher relevance to the user query. As shown in Table 1, it can be observed that WCM-based algorithms provide high accuracy in terms of relevancy, but they take more time since they compare the user query to the content of the page, such as Query-Dependent algorithm and SimRank algorithm. The Tag Rank algorithm provides low relevancy with less execution time because it compares the user query with the keyword associated with the web pages rather than the content of the web page. In order to provide higher result relevancy with lower time complexity, I believe it is preferable to combine more than one algorithm to produce a hybrid ranking algorithm.

\section{CONCLUSION}

Generally, to explore information on the internet, users utilize search engines. Users submit the query to obtain results that are retrieved by the search engine. In order to decide which page to be presented at the beginning of the search results, different algorithms for ranking the web pages are employed by the search engines. This paper 
conducted an analysis of the existing algorithms in ranking web pages. In addition, a comparison of the various algorithms was done based on multiple parameters, such as web mining technique, and algorithm methodology, as well as input parameters, and the relevancy of the result to a user's query. Finally, as shown in Table 1, among all 12 ranking algorithms, it can be observed that the algorithms that perform better in terms of relevancy were Query-Dependent, SimRank, Relation Based, and Time Rank algorithms. While WPR, HITS, EigenRumor, and Tag Rank were the algorithms that perform better in terms of time complexity.

\section{REFERENCES}

[1] U. Naik and D. Shivalingaiah, "Comparative Study of Web 1. 0, Web 2. 0 and Web 3. 0," 6th International CALIBER, pp. 499-507, 2008 .

[2] K. Jacksi and S. M. Abass, "Development history of the world wide web," Int. J. Sci. Technol. Res, vol. 8, no. 9, Art. no. 9, 2019.

[3] R. KumarRana and N. Tyagi, "A Novel Architecture of Ontologybased Semantic Web Crawler," International Journal of Computer Applications, vol. 44, no. 18, pp. 31-36, 2012, doi: 10. 5120/63658724.

[4] M. A. Sadeeq, S. R. Zeebaree, R. Qashi, S. H. Ahmed, and K Jacksi, "Internet of Things security: a survey," 2018, pp. 162-166.

[5] K. Jacksi, N. Dimililer, and S. Zeebaree, "State of the art exploration systems for linked data: a review," Int. J. Adv. Comput. Sci. Appl. IJACSA, vol. 7, no. 11, pp. 155-164, 2016

[6] K. Jacksi, N. Dimililer, and S. R. Zeebaree, "A survey of exploratory search systems based on LOD resources," 2015.

[7] K. Jacksi, "Design And Implementation Of Online Submission And Peer Review System: A Case Study Of E-Journal Of University Of Zakho," International Journal of Scientific \& Technology Research, vol. 4, no. 8, pp. 83-85, 2015.

[8] M. P. S. M. E, "Ranking Techniques for Social Networking Sites based on Popularity," Indian Journal of Computer Science and Engineering (IJCSE) Ranking, vol. 3, no. 3, pp. 522-526, 2012.

[9] J. Cho, S. Roy, and R. E. Adams, "Page quality: In search of an unbiased web ranking," Proceedings of the ACM SIGMOD International Conference on Management of Data, pp. 551-562, 2005, doi: 10. 1145/1066157. 1066220.

[10] K. Jacksi, S. Zeebaree, and N. Dimililer, "Design and Implementation of LOD Explorer: A LOD Exploration and Visualization Model," Journal of Applied Science and Technology Trends, vol. 1, no. 2, pp. 31-39, 2020.

[11] A. Barbar and A. Ismail, "Search engine optimization (SEO) for websites," ACM International Conference Proceeding Series, vol. Part F1482, pp. 51-55, 2019, doi: 10. 1145/3323933. 3324072.

[12] K. Jacksi and S. Badiozamany, "General method for data indexing using clustering methods," Int. J. Sci. Eng, vol. 6, no. 3, pp. 641644, 2015.

[13] S. R. Z. Karwan Jacksi Nazife Dimililer, "AN IMPROVED APPROACH FOR INFORMATION RETRIEVAL WITH SEMANTIC-WEB CRAWLING," University of Zakho, 2018.

[14] N. Grover and R. Wason, "Comparative Analysis Of Page Rank And HITS Algorithms," International Journal of Engineering Research \& Technology (IJERT), vol. 1, no. 8, pp. 1-15, 2012.

[15] D. Mukhopadhyay, P. Biswas, and Y. -C. Kim, "A Syntactic Classification based Web Page Ranking Algorithm," pp. 83-92, 2011.

[16] M. PaulSelvan, A. Chandra Sekar, and A. Priya Dharshini, "Survey on Web Page Ranking Algorithms," International Journal of Computer Applications, vol. 41, no. 19, pp. 1-7, 2012, doi: 10. 5120/5646-7764.

[17] P. S. Sharma, D. Yadav, and P. Garg, "A systematic review on page ranking algorithms," International Journal of Information Technology (Singapore), vol. 12, no. 2, pp. 329-337, 2020, doi: 10. 1007/s41870-020-00439-3.
[18] P. Desikan, J. Srivastava, V. Kumar, and P. Tan, "Hyperlink analysis : techniques and applications," p. 42, 2002.

[19] D. Kumar Sharma and A. K. Sharma, "A Comparative Analysis of Web Page Ranking Algorithms," IJCSE) International Journal on Computer Science and Engineering, vol. 02, no. 08, pp. 2670-2676, 2010.

[20] et al Srivastava, Jaideep, "Web Usage Mining: Discovery and Applications of Usage Patterns from Web Data INTRODUCTION," ACM SIGKDD Explorations Newsletter 1. 2, vol. 1, no. 2, pp. 12-23, 2011.

[21] R. Ibrahim, S. Zeebaree, and K. Jacksi, "Survey on Semantic Similarity Based on Document Clustering," Adv. sci. technol. eng. syst. j, vol. 4, no. 5, pp. 115-122, 2019.

[22] K. Jacksi, R. Kh. Ibrahim, S. R. M. Zeebaree, R. R. Zebari, and M. A. M. Sadeeq, "Clustering Documents based on Semantic Similarity using HAC and K-Mean Algorithms," in 2020 International Conference on Advanced Science and Engineering (ICOASE), Dec. 2020, pp. 205-210. doi: 10. 1109/ICOASE51841. 2020. 9436570

[23] S. M. Mohammed, K. Jacksi, and S. Zeebaree, “A state-of-the-art survey on semantic similarity for document clustering using GloVe and density-based algorithms," Indonesian Journal of Electrical Engineering and Computer Science, vol. 22, no. 1, pp. 552-562, 2021.

[24] S. M. Mohammed, K. Jacksi, and S. R. M. Zeebaree, "Glove Word Embedding and DBSCAN algorithms for Semantic Document Clustering," in 2020 International Conference on Advanced Science and Engineering (ICOASE), Dec. 2020, pp. 1-6. doi: 10. 1109/ICOASE51841. 2020. 9436540.

[25] N. M. Salih and K. Jacksi, "State of the art document clustering algorithms based on semantic similarity," JURNAL INFORMATIKA, vol. 14, no. 2, pp. 58-75, 2020

[26] N. M. Salih and K. Jacksi, "Semantic Document Clustering using K-means algorithm and Ward's Method," in 2020 International Conference on Advanced Science and Engineering (ICOASE), Dec. 2020, pp. 1-6. doi: 10. 1109/ICOASE51841. 2020. 9436588.

[27] P. Gupta, S. K. Singh, D. Yadav, and A. K. Sharma, “An improved approach to ranking web documents," Journal of Information Processing Systems, vol. 9, no. 2, pp. 217-236, 2013, doi: 10. 3745/JIPS. 2013. 9. 2. 217.

[28] L. Jin, L. Feng, G. Liu, and C. Wang, "Personal Web Revisitation by Context and Content Keywords with Relevance Feedback," IEEE Transactions on Knowledge and Data Engineering, vol. 29, no. 7, pp. 1508-1521, 2017, doi: 10. 1109/TKDE. 2017. 2672747.

[29] P. Gupta, A. K. Sharma, and D. Yadav, "A NOVEL TECHNIQUE FOR BACK-LINK EXTRACTION AND RELEVANCE EVALUATION," Int. J. Comput. Sci. Inf. Technol., vol. 3, no. 3, pp. 227-238, 2011.

[30] W. Xing and A. Ghorbani, "Weighted PageRank algorithm," Proceedings - Second Annual Conference on Communication Networks and Services Research, pp. 305-314, 2004, doi: 10. 1109/dnsr. 2004. 1344743.

[31] J. M. Kleinberg, "Authoritative sources in a hyperlinked environment," Proceedings of the ninth annual ACM-SIAM symposium on Discrete algorithms, vol. 9781400841, no. May 1997, pp. 668-677, 1998, doi: 10. 1515/9781400841356. 514.

[32] K. Fujimura, T. Inoue, and M. Sugisaki, "The EigenRumor Algorithm for Ranking Blogs," Proceedings of the Second Annual Workshop on the Weblogging Ecosystem: Aggregation, Analysis and Dynamics, Chiba, Japan, pp. 59-74, 2005.

[33] A. M. ZarehBidoki and N. Yazdani, "DistanceRank: An intelligent ranking algorithm for web pages," Information Processing and Management, vol. 44, no. 2, pp. 877-892, 2008, doi: 10. 1016/j. ipm. 2007. 06. 004.

[34] L. W. Lee, J. Y. Jiang, C. Der Wu, and S. J. Lee, "A querydependent ranking approach for search engines," 2 nd International Workshop on Computer Science and Engineering, WCSE 2009, vol. 1, pp. 259-263, 2009, doi: 10. 1109/WCSE. 2009. 666.

[35] C. Li et al. , "Fast computation of SimRank for static and dynamic information networks," Advances in Database Technology - EDBT 2010 - 13th International Conference on Extending Database Technology, Proceedings, pp. 465-476, 2010, doi: 10. 1145/1739041. 1739098.

[36] R. Baeza-Yates and E. Davis, "Web Page Ranking using Link Attributes Categories and Subject Descriptors," Proceedings of the 
13th international World Wide Web conference on Alternate track papers \& posters, pp. 328-329, 2004.

[37] F. Lamberti, A. Sanna, and C. Demartini, "A relation-based page rank algorithm for semantic Web search engines," IEEE Transactions on Knowledge and Data Engineering, vol. 21, no. 1, pp. 123-136, 2009, doi: 10. 1109/TKDE. 2008. 113.

[38] D. H. Maulud, S. R. Zeebaree, K. Jacksi, M. A. M. Sadeeq, and K. H. Sharif, "State of art for semantic analysis of natural language processing," Qubahan Academic Journal, vol. 1, no. 2, pp. 21-28, 2021

[39] K. J. A Zeebaree SRM Zeebaree, "Designing an Ontology of Elearning system for Duhok Polytechnic University Using Protégé OWL Tool," J. Adv. Res. Dyn. Control Syst. , vol, vol. 11, no. 5, pp. 24-37, 2019.

[40] S. R. M. Z. Adel AL-Zebari Karwan Jacksi and Ali Selamat, "ELMS-DPU Ontology Visualization with Protégé VOWL and Web VOWL," Journal of Advanced Research in Dynamical and Control Systems, vol. 11, no. 1, pp. 478-485, 2019.

[41] A. -Z. Adel, S. Zebari, and K. Jacksi, "Football Ontology Construction using Oriented Programming," Journal of Applied Science and Technology Trends, vol. 1, no. 1, pp. 24-30, 2020.

[42] K. Jacksi, "Design and Implementation of E-Campus Ontology with a Hybrid Software Engineering Methodology," Science Journal of University of Zakho, vol. 7, no. 3, pp. 95-100, 2019.

[43] S. Jie, C. Chen, Z. Hui, S. Rong-Shuang, Z. Yan, and H. Kun, "Tag Rank: A new rank algorithm for webpage based on social web," Proceedings of the International Conference on Computer Science and Information Technology, ICCSIT 2008, pp. 254-258, 2008, doi: 10. 1109/ICCSIT. 2008. 45

[44] H. Jiang, Y. X. Ge, D. Zuo, and B. Han, "TimeRank: A method of improving ranking scores by visited time," Proceedings of the 7th International Conference on Machine Learning and Cybernetics, ICMLC, vol. 3, pp. 1654-1657, 2008, doi: 10. 1109/ICMLC. 2008. 4620671 . 